\title{
TOWARDS A FORMAL MODEL OF CyberRail
}

\author{
Dines Bjørner ${ }^{1,2}$ \\ (1) Department of Computer Science, School of Computing, National University of Singapore, 3 \\ Science Drive 2, Singapore 117543 \\ E-Mail: bjorner@comp.nus.edu.sg, URL: http://www.comp.nus.edu.sg/cs/
}

Peter Chiang ${ }^{2}$, Morten S.T. Jacobsen ${ }^{2}$, Jens Kielsgaard Hansen², and Michael P. Madsen ${ }^{2}$

(2) Section of Computer Science and Engineering, Institute of Informatics and Computer Engineering, Technical University of Denmark, DK-2800 Kgs.Lyngby, Denmark

E-Mail: \{s001402,s001693,s001842,s001700\}@student.dtu.dk

\section{Martin Penicka ${ }^{1,3 *}$}

(3) Czech Technical University, Faculty of Transportation Sciences, Department of Applied Mathematics, Na Florenci 25, 11000 Praha 1, Czech Republic

E-Mail: penicka@fd.cvut.cz

\begin{abstract}
Based on a number of reports and publications, primarily by Takahiko Ogino [14], [15], [16] (in these proceedings), and [17], on the emerging concept of CyberRail, we attempt to show what a formal domain model of CyberRail might look like, and what benefits one might derive from establishing and having such a formal model.
\end{abstract}

Keywords: CyberRail, Formalisation, Domain Model, RAISE, RSL, CSP, Non-determinism, Abstract Data Types

\section{Background}

The background for the work reported in this extended abstract is threefold: (i) Many years of actual formal specification as well as research into how to engineer such formal specifications, by the first author, of domains, including the railway domain [8] [7] [9] [10] [1] [6] [3] [2] [5] —using abstraction and

\footnotetext{
*MPs work was supported, in part, by the EU 5th IST FP Programme acttion: CoLogNET: The Computational Logic Network of Excellence.
} 
modelling principles and techniques extensively covered in three forthcoming software engineering textbooks [4]. (ii) A term project with four MSc students. And (iii) Some fascination as whether one cold formalise an essence of the novel ideas of CyberRail. We strongly believe that we can capture one crucial essence of CyberRail — such as this paper will show.

The formalisation of CyberRail is expressed in the RAISE [12] Specification Language, RSL [11]. RAISE stands for Rigorous Approach to Industrial Software Engineering. In the current abstract model we especially make use of RSL's parallel process modeling capability. It builds on, ie., borrows from Tony Hoare's algebraic process concept of Communicating Sequential Processes, CSP [13].

\section{A Rough Sketch Formal Model}

\subsection{An Overall CyberRail System}

CyberRail consists of an index set of traveller behaviours and one cyber behaviour "running" in parallel. Each traveller behaviour is uniquely identified, p:Tx. Traveller behaviours communicate with the cyber behaviour. We abstract the communication medium as an indexed set of channels, ct[p], from the cyber behaviour to each individual traveller behaviour, and tc[p], from traveller behaviours to the cyber behaviour. Messages over channels are of respective types, CT and TC. The cyber behaviour starts in an initial state $\omega_{i}$, and each traveller behaviour, $p$, starts in some initial state $m \sigma_{i}(p)$.

\section{type}

Tx, $\Sigma, \Omega, \mathrm{CT}, \mathrm{TC}$

$\mathrm{M} \Sigma=\mathrm{Tx} \underset{\mathrm{m}}{\mathrm{N}} \Sigma$

channel

value

$\{\mathrm{ct}[\mathrm{p}]: \mathrm{CT}, \mathrm{tc}[\mathrm{p}]: \mathrm{TC} \mid \mathrm{p}: \mathrm{Tx}\}, \mathrm{cr}: \mathrm{CR}, \mathrm{rc}: \mathrm{RC}$

$\mathrm{m} \sigma_{i}: \mathrm{M} \Sigma, \omega_{i}: \Omega$

cyberrail_system: Unit $\rightarrow$ Unit

cyberrail_system ()$\equiv\left\|\left\{\operatorname{traveller}(\mathrm{p})\left(\mathrm{m} \sigma_{i}(\mathrm{p})\right) \mid \mathrm{p}: \operatorname{Tx}\right\}\right\| \operatorname{cyber}(\omega)$

cyber: $\Omega \rightarrow$ in $\{\mathrm{tc}[\mathrm{p}] \mid \mathrm{p}: \mathrm{Tx}\}$, cr out $\{\mathrm{ct}[\mathrm{p}] \mid \mathrm{p}: \mathrm{Tx}\}, \mathrm{rc}$ Unit

$\operatorname{cyber}(\omega) \equiv$

cyber_as_server $(\omega) \prod$ cyber_as_proactive $(\omega) \prod$ cyber as co director $(\omega)$

traveller: $\mathrm{p}: \mathrm{Tx} \rightarrow \Sigma \rightarrow$ in $\operatorname{ct}[\mathrm{p}]$ out tc $[\mathrm{p}]$ Unit $\operatorname{traveller}(\mathrm{p})(\sigma) \equiv$ active_traveller(p) $(\sigma) \prod$ passive $\operatorname{traveller}(\mathrm{p})(\sigma)$ 
The cyber behaviour either acts as a server: Ready to engage in communication input from any traveller behaviour; or the cyber behaviour acts proactively: Ready to engage in performing output to one, or some traveller behaviours; or the cyber behaviour acts in consort with the "rest" of the transportation market (including rail infrastructure owners, train operators, etc.), in improving and changing services, and in otherwise responding to unforeseen circumstances of that market.

Similarly any traveller behaviour acts as a client: Ready to engage in performing output to the cyber behaviour; or its acts passively: Ready to accept input from the cyber behaviour.

\subsection{Travellers}

2.2.1 Active Travellers. Active traveller behaviours alternate internally non-deterministically, ie., at their own choice, between start (travel) planning st_pl, select (among suggested) travel plan(s) se_pl, change (travel) planning ch_pl, begin travel be_tr, board train bo_tr, leave train $l v \_t r$, ignore train ig_tr, cancel travel ca_tr, seeking guidance se_gu, notifying cyber no_cy, entertainment ent, deposit resource de_re (park car, ...), claim resource cl_re (retreive car, ...), get resource ge_re (rent a car, ...), return resource re_re (return rent-car, ...), going to restaurant rest (or other), change travel ch_tr, interrupt travel in_tr, resume travel re_tr, leave train le_tr, end travel en_tr, and many other choices. Each of these normally entail an output communication to the cyber behaviour, and for those we can assume immediate response from the cyber behaviour, where applicable.

\section{value}

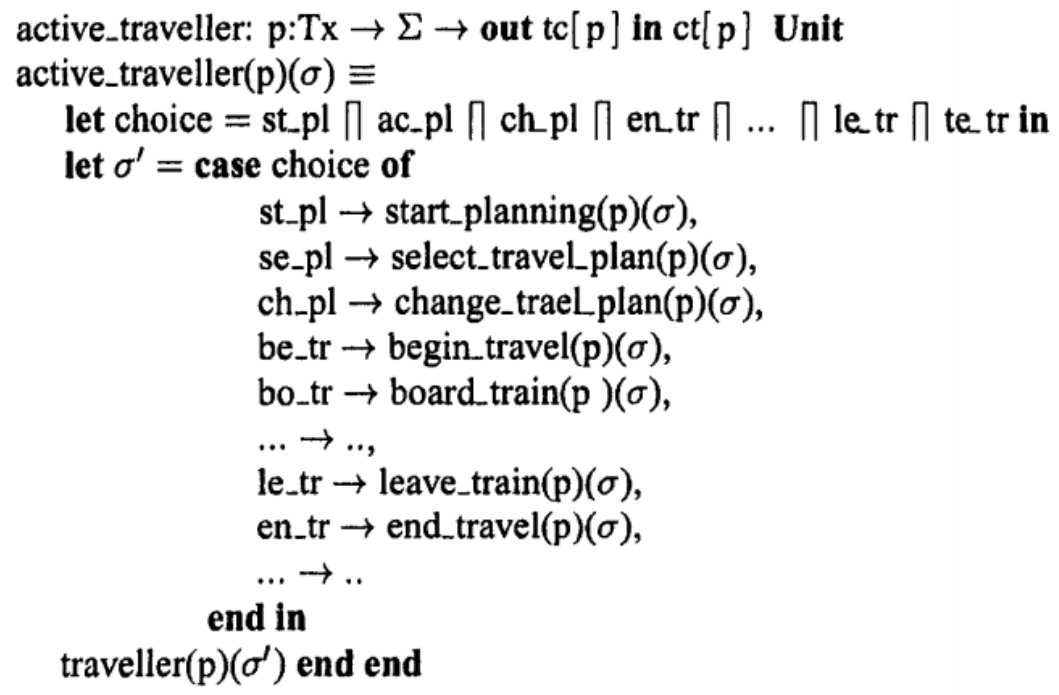




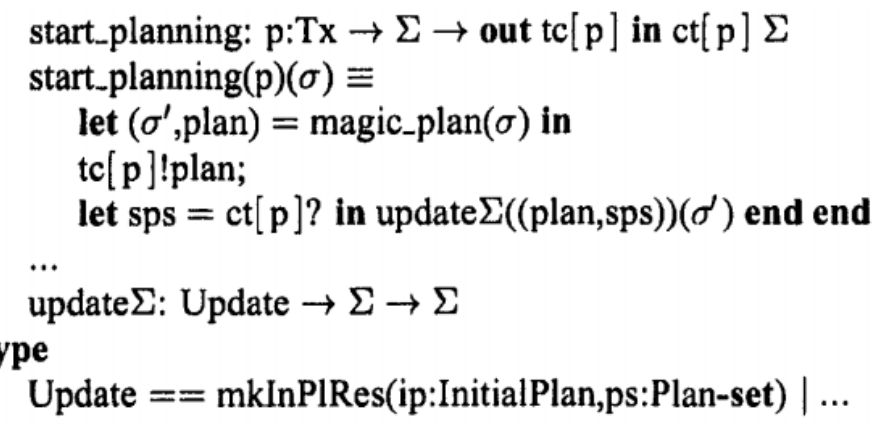

2.2.2 Passive Travellers. When not engaging actively with the cyber behaviour, traveller behaviours are ready to accept any cyber initated action. The traveller behaviour basically "assimilates" messages received from cyber — and may make use of these in future.

\section{value}

passive_traveller: $\mathrm{p}: \mathrm{Tx} \rightarrow \Sigma \rightarrow$ in ct $[\mathrm{p}]$ out tc[p] Unit

passive_traveller(p) $(\sigma) \equiv$ let res $=\operatorname{ct}[\mathrm{p}]$ ? in update $\Sigma(\mathrm{res})(\sigma)$ end

2.2.3 Active Traveller Actions. The active_traveller behaviour performs either of the internally non-deterministically chosen actions: start_planning, select_travel_plan, change_travel_plan, begin_travel, board_train, ..., leave_train, or end_travel. They make use only of the "sum total state" $(\sigma)$ that that traveller behaviour "is in". Each such action basically communicates either of a number of plans (or parts thereof, here simplified into plans). Let us summarise:

type

Plan

Request $=$ Initial_Plan $\mid$ Selected_Plan $\mid$ Change_Plan $\mid$ Begin Travel

Initial_Plan $==$ mkIniPl(pl:Plan) $\mid$ Board_Train $|\ldots|$ Leave_Train | End_Travel $\mid \ldots$

Selected_Plan $==\operatorname{mkSelPl}($ pl:Plan)

Change_Plan $==\operatorname{mkChgPl}($ pl:Plan $)$

Begin_Travel $==\operatorname{mkBTrav}(\mathrm{pl}:$ Plan)

Board_Train $==\operatorname{mkBTrai}(\mathrm{pl}:$ Plan $)$

Leave_Train $==$ mkLeTr(pl:Plan)

End_Travel $==\operatorname{mkEnTr}($ pl:Plan $)$

value

$\forall \mathrm{f}: \mathrm{p}: \mathrm{Tx} \rightarrow \Sigma \rightarrow$ out tc $[\mathrm{p}] \Sigma$ 


\section{magic_f: $\Sigma \rightarrow \Sigma \times$ Request}

$$
\mathrm{f}(\mathrm{p})(\sigma) \equiv \text { let }\left(\sigma^{\prime}, \text { req }\right)=\text { magic_f }(\sigma) \text { in tc }[\mathrm{p}] ! \text { req; } \sigma^{\prime} \text { end }
$$

The magic_functions access and changes the state while otherwise yielding some request. They engage in no events with other than the traveller state. There are the possibility of literally "zillions" such functions, all fitted into the above sketched traveller behaviour.

\section{3 cyber}

2.3.1 cyber as Server. cyber is at any moment ready to engage in actions with any traveller behaviour. cyber is assumed here to respond immediately to "any and such".

\section{value}

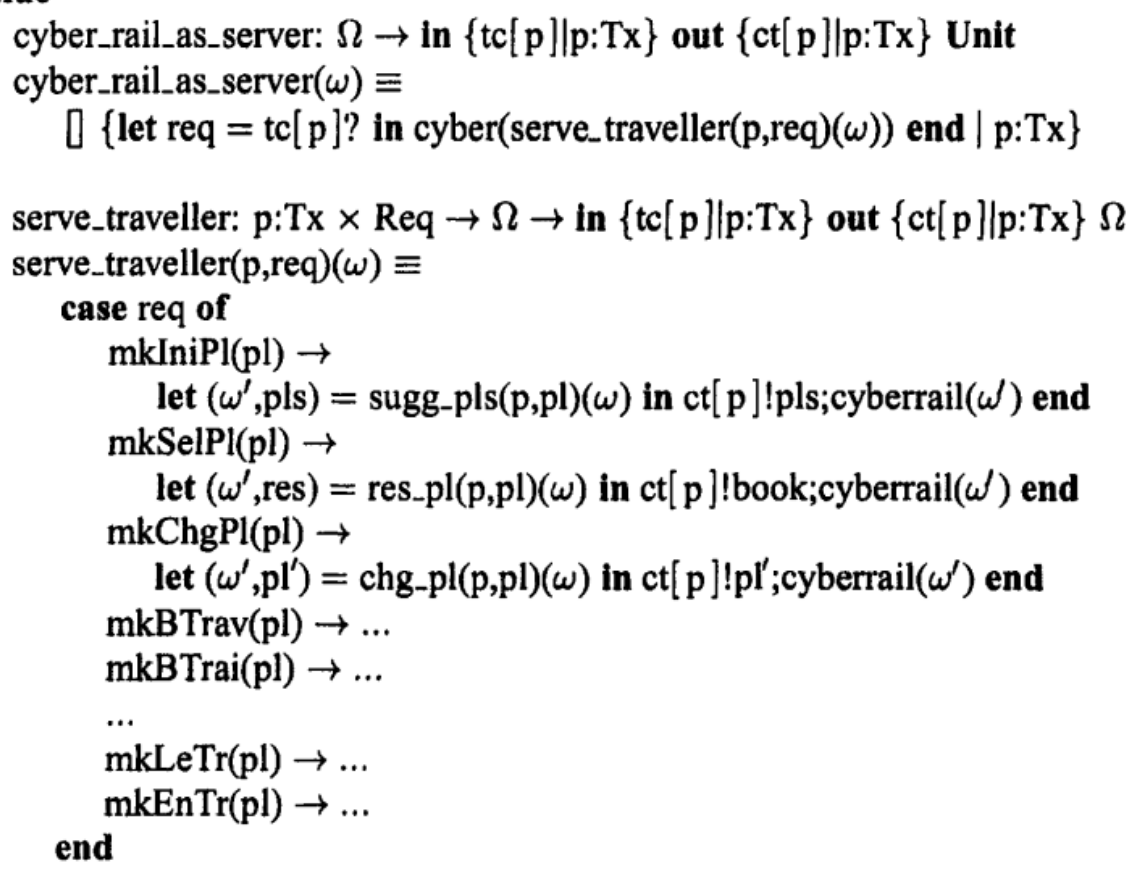

2.3.2 cyber as Pro-Active. cyber, on its own volition, may, typically based on its accumulated knowledge of traveller behaviours, engage in sending messages of one kind or another to selected groups of travellers. Section 2.3.5 rough sketch-formalises one of these.

\section{type}

CR_act $==$ gu_tr $\mid$ no_tr $\mid$ co_tr $\mid$ wa_tr $\mid \ldots$ 


\section{value}

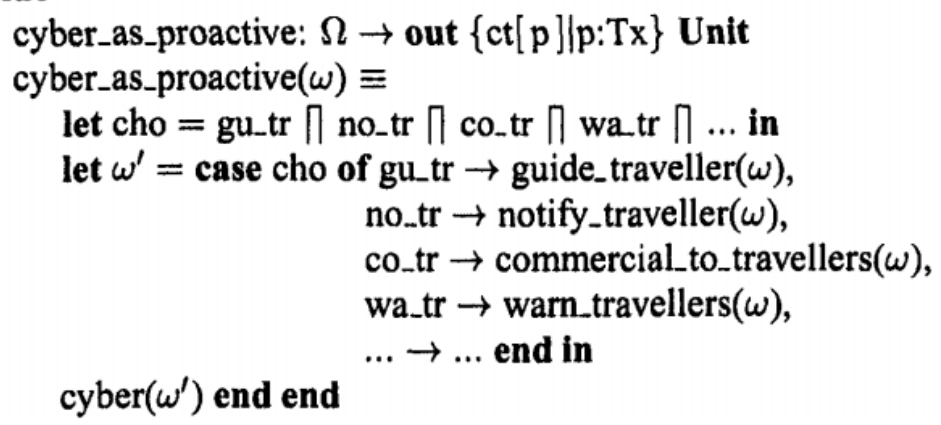

2.3.3 cyber as Co-Director. We do not specify this behaviour. It concerns the actions that cyber takes together with the "rest" of the transportation market. One could mention input from cyber_as_co_director to the train operators as to new traveller preferences, profiles, etc., and output from the rail (ie., net) infrastructure owners or train operators to cyber_as_co_director as to net repairs or train shortages, etc. The decomposition of CyberRail into cyber and the "rest", may - to some - be articificial, namely in countries where there is no effective privatisation and split-up into infrastructyre owners and train operators. But it is a decomposition which is relevant, structurally, in any case.

2.3.4 cyber Server Actions. We sketch:

value

$$
\begin{aligned}
& \text { sugg_plans: } \mathrm{p}: \mathrm{Tx} \times \text { Plan } \rightarrow \Omega \rightarrow \Omega \times \text { Plan-set } \\
& \text { res_pl: p:Tx } \times \text { Plan } \rightarrow \Omega \rightarrow \Omega \times \text { Plan } \\
& \text { chg_pl: p:Tx } \times \text { Plan } \rightarrow \Omega \rightarrow \Omega \times \text { Plan }
\end{aligned}
$$

There are many other such traveller instigated cyber actions.

2.3.5 Pro-Active cyber Actions. We rough sketch just a single of the possible "dozens" of cyber inititated actions versus the travellers.

\section{value}

guide_traveller: $\Omega \rightarrow$ out $\{\mathrm{ct}[\mathrm{p}] \mid \mathrm{p}: \mathrm{Tx}\} \Omega$

guide_traveller $(\omega) \equiv$

let $\left(\omega^{\prime},(\mathrm{ps}\right.$, guide $\left.)\right)=$ any_guide $(\omega)$ in broadcast(ps,guide $) ; \omega$ end

any_guide: $\Omega \rightarrow \Omega \times($ Tx-set $\times$ Guide $)$

notify_traveller: $\Omega \rightarrow$ out $\{\operatorname{ct}[\mathrm{p}] \mid \mathrm{p}: \mathrm{Tx}\} \Omega$ 
commercial_to_travellers: $\Omega \rightarrow$ out $\{\operatorname{ct}[\mathrm{p}] \mid \mathrm{p}: \mathrm{Tx}\} \Omega$

warn_traveller: $\Omega \rightarrow$ out $\{\mathrm{ct}[\mathrm{p}] \mid \mathrm{p}: \mathrm{Tx}\} \Omega$

$\cdots$

\author{
broadcast: Tx-set $\times \mathrm{CT} \rightarrow$ Unit \\ broadcast(ps,msg) $\equiv$ \\ case ps of \{\}$\rightarrow$ skip, $\{p\} \cup p s^{\prime} \rightarrow c t[p] ! m s g ;$ broadcast(ps',msg) end
}

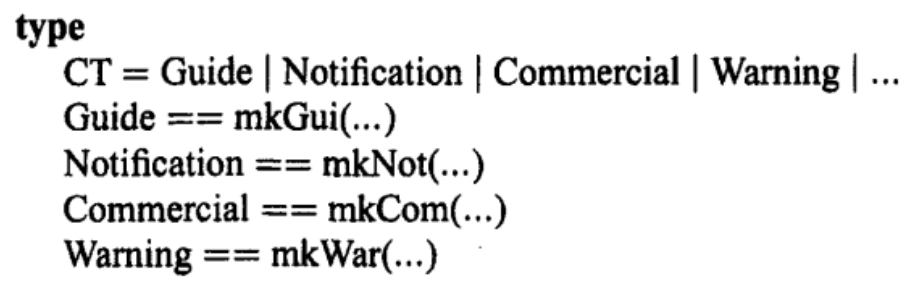

\title{
3. Conclusion
}

A formalisation of a crucial aspect of CyberRail has been sketched. Namely the interplay between the rôles of travellers and the central CyberRail system.

Next we need analyse carfully all the action functions with respect to the way in which they use and update the respective states $(\sigma: \Sigma)$ of traveller behaviours and the cyber behaviour $(\omega: \Omega)$. At the end of such an analysis one can then come up with precise, formal descriptions, including axioms, of what the title of [16] refers to as the Information Infrastructure. We look forward to report on that in a near future.

The aim of this work is to provide a foundation, a domain theory, for CyberRail. A set of models from which to "derive", in a systematic way, proposals for computing systems, including software architectures.

\section{References}

[1] Dines Bjørner. Formal Software Techniques in Railway Systems. In Eckehard Schnieder, editor, 9th IFAC Symposium on Control in Transportation Systems, pages 1-12, Braunschweig, Germany, 13-15 June 2000. Invited talk.

[2] Dines Bjørner. Dynamics of Railway Nets: On an Interface between Automatic Control and Software Engineering. In CTS2003: 10th IFAC Symposium on Control in Transportation Systems, Oxford, UK, August 4-6 2003. Elsevier Science Ltd.

[3] Dines Bjørner. New Results and Trends in Formal Techniques for the Development of Software for Transportation Systems. In FORMS2003: Symposium on Formal Methods for Railway Operation and Control Systems. 2003. Budapest, Hungary. Editors: G. Tarnai and E. Schnieder, Germany. 
[4] Dines Bjørner. Software Engineering, volume Vol. 1: Abstraction and Modelling, Vol. 2: Advanced Specification Techniques, Vol. 3: From Domains via Requirements to Software. Springer-Verlag, 2004-2005.

[5] Dines Bjørner, Chris George, Anne E. Haxthausen, Christian Krog Madsen, Steffen Holmslykke, and Martin Ponioka. "UML"-ising Formal Techniques. In INT 2004: Third International Workshop on Integration of Specification Techniques for Applications in Engineering. 28 March 2004, ETAPS, Barcelona, Spain. INT-2004 Proceedings, SpringerVerlag.

[6] Dines Bjørner, Chris W. George, and Søren Prehn. Computing Systems for Railways A Rôle for Domain Engineering. Relations to Requirements Engineering and Software for Control Applications. In Integrated Design and Process Technology. Editors: Bernd Kraemer and John C. Petterson, 24-28 June 2002. Society for Design and Process Science.

[7] Dines Bjørner, C.W. George, and S. Prehn. Scheduling and Rescheduling of Trains, chapter 8, pages 157-184. Industrial Strength Formal Methods in Practice, Eds.: Michael G. Hinchey and Jonathan P. Bowen. FACIT, Springer-Verlag, London, England, 1999.

[8] Dines Bjørner, Dong Yu Lin, and S. Prehn. Domain Analyses: A Case Study of Station Management. In KICS'94: Kunming International CASE Symposium, Yunnan Province, P.R.ofChina. Software Engineering Association of Japan, 16-20 November 1994.

[9] Dines Bjørner, Søren Prehn, and Chris W. George. Formal Models of Railway Systems: Domains. FME Rail Workshop on Formal Methods in Railway Systems, FM'99 World Congress on Formal Methods, France.

[10] Dines Bjørner, Søren Prehn, and Chris W. George. Formal Models of Railway Systems: Requirements. FMERail Workshop on Formal Methods in Railway Systems, FM'99 World Congress on Formal Methods, France.

[11] Chris George, Peter Haff, Klaus Havelund, Anne Haxthausen, Robert Milne, Claus Bendix Nielsen, Søren Prehn, and Kim Ritter Wagner. The RAISE Specification Language. The BCS Practitioner Series. Prentice-Hall, Hemel Hampstead, England, 1992.

[12] Chris George, Anne Haxthausen, Steven Hughes, Robert Milne, Søren Prehn, and Jan Storbank Pedersen. The RAISE Method. The BCS Practitioner Series. Prentice-Hall, Hemel Hampstead, England, 1995.

[13] C.A.R. Hoare. Communicating Sequential Processes. C.A.R. Hoare Series in Computer Science. Prentice-Hall International, 1985.

[14] Takahiko Ogino. Aiming for Passenger Interoperability. Technical report, Railway Technical Research Institute, Transport Information Technology Division, Railway Technical Research Institute, 2-8-38 Hikari-cho, Kokubunji-shi, Tokyo, 185-8540 Japan, 2003.

[15] Takahiko Ogino. CyberRail: For Urban Mobility Tomorrow. Technical report, Railway Technical Research Institute, Transport Information Technology Division, Railway Technical Research Institute, 2-8-38 Hikari-cho, Kokubunji-shi, Tokyo, 185-8540 Japan, 2004.

[16] Takahiko Ogino. CyberRail: Information Infrastructure for New Intermodal Transport Business Model. In Topical Days @ IFIP World Computer Congress 2004, IFIP Series. IFIP, Kluwer Academic Press, August 2004.

[17] Takahiko Ogino, Koivhi Goto, Ryuji Tsuchiya, Kiyotaka Seki, and Akihiko Matsuoka. CyberRail and its significance in the coming ubiquitous society. In , 2004. 